\title{
Role of cardiac nuclear stress perfusion exam after computed tomographic coronary angiogram for evaluation of obstructive coronary artery disease in patients with chest pain
}

\author{
Maryam Gul ${ }^{1}$, Mubashir Sheikh ${ }^{2}$, Abbas Chaudhry ${ }^{2}$, Luke Gerges ${ }^{2}$, Hadi Al Halabi ${ }^{2}$ Eric Feldman ${ }^{3}$, \\ Ammar Chaudhry ${ }^{3}$ \\ ${ }^{1}$ Precision Rheumatology, Anaheim, CA, USA; ${ }^{2}$ Department of Diagnostic and Interventional Radiology, City of Hope National Medical Center, \\ Duarte, CA, USA; ${ }^{3}$ Department of Diagnostic and Interventional Radiology, Stony Brook University Medical Center, Stony Brook, NY, USA \\ Contributions: (I) Conception and design: E Feldman, A Chaudhry; (II) Administrative support: M Gul, E Feldman, A Chaudhry; (III) Provision of \\ study materials or patients: E Feldman, A Chaudhry; (IV) Collection and assembly of data: L Gerges, E Feldman, A Chaudhry; (V) Data analysis and \\ interpretation: L Gerges, M Gul, A Chaudhry; (VI) Manuscript writing: All authors; (VII) Final approval of manuscript: All authors. \\ Correspondence to: Dr. Ammar Chaudhry, MD. 1500 E. Duarte Rd., Duarte, CA 91010, USA. Email: achaudhry@coh.org.
}

Background: Clinical workup for chest pain varies among institutions. Acute coronary syndrome (ACS) is the primary diagnosis to rule out in the differential diagnosis, due to its associated mortality and morbidity. Although studies have demonstrated efficacy of coronary computed tomographic angiography (CCTA) in diagnosis obstructive coronary artery disease (CAD), there is limited evidence in the clinical value of performing cardiac nuclear stress perfusion imaging [myocardial perfusion imaging (MPI)] exam in patients with chest pain after undergoing CCTA. We aim to evaluate clinical value of follow-up nuclear cardiac MPI in patients with chest pain who have undergone recent CCTA.

Methods: A total of 1,000 patients were evaluated in this IRB approved retrospective study who presented with symptoms of ACS. Patients who had elevated troponin or abnormal electrocardiogram (ECG) findings at initial presentation or prior to cardiac nuclear MPI were excluded from the study. All patients who underwent 64- or 320-detector row ECG-gated CCTA as well as a follow-up nuclear MPI. Patients who had diagnostics studies limited by artifact [e.g., suboptimal intravenous (IV) contrast bolus in CCTA, motion artifact on CCTA or MPI, etc.] were excluded.

Results: One hundred patients met the inclusion criteria. Patient demographics include average age 64.3 [32-89] years, 59 male, 41 females. Ninety-five/100 patients had at least one vessel with 50-70\% coronary artery diameter stenosis measured on CCTA. There were no focal perfusion abnormalities identified on cardiac nuclear MPI in patients with less than $70 \%$ stenosis diagnosed on CCTA. Five percent of patients were identified with coronary arterial narrowing greater than $70 \%$ on CCTA and all 5 of these patients have evidence of abnormal cardiac nuclear stress test (perfusion abnormalities, chest pain, abnormal ECG).

Conclusions: In low-to-intermediate risk patients with chest pain and evidence of non-critical coronary artery stenosis (i.e., less than $70 \%$ stenosis) diagnosed on CCTA, a follow-up cardiac nuclear perfusion imaging is of limited value.

Keywords: Coronary computed tomographic angiogram (CCTA); nuclear myocardial perfusion imaging (nuclear $\mathrm{MPI})$; acute coronary syndrome (ACS); chest pain

Submitted Apr 01, 2020. Accepted for publication May 06, 2020.

doi: $10.21037 /$ jtd-2019-pitd-12

View this article at: http://dx.doi.org/10.21037/jtd-2019-pitd-12 


\section{Introduction}

Chest pain is one of the most common (\#2) cause of patient visits to the emergency department (ED) in USA (1-5). Various studies have shown that frequently (greater than $50 \%$ of time), a non-cardiac condition is the reason for acute chest pain. However, ACS can have atypical clinical presentations and ACS symptoms can overlap with non-cardiac etiologies. This makes rapid and accurate diagnosis of the chest pain cause quite challenging (2-4). Currently, most patient care facilities across the nation utilize the following "rule-out myocardial infarction (MI)" diagnostic algorithm: point of care troponin plus electrocardiogram (ECG). If initial tests are negative, then depending on patients' risk factors, patient may undergo serial ECG and cardiac enzymes along with coronary computed tomographic angiogram (CCTA) and/or stress cardiac nuclear myocardial perfusion imaging (MPI) $(4,5)$. Although this approach has reduced the number of major diagnostic errors, but it has shown to increase the length of stay, and cost of care in the ED and the result is not always definitive (5). Moreover, side effects such as dyspnea, tachycardia, and flushing are very common with widely used nuclear stress test chemical agents such as Adenosine and Regadenon (6). Recent studies have shown CCTA can be a rapid, noninvasive, and less expensive test with a high negative predictive value (95-99\%) that reduces the length of stay in low to intermediate risk patients with possible ACS (7-11). However, some studies suggest that CCTA may increase the rate of invasive coronary angiography procedures and revascularizations with no significant difference in the length of stay (8-12). Also, at present patient with "non-critical" (less than $90 \%$ stenosis) and/or "borderline obstructive" 50-69\% diameter stenosis frequently undergo further diagnostic work-up, e.g., nuclear cardiac MPI (9-12). The purpose of this study was to evaluate clinical value of follow-up nuclear cardiac MPI in patients with chest pain who have undergone recent CCTA revealing noncritical (less than $70 \%$ diameter) stenosis. We present the following article in accordance with the STROBE reporting checklist (available at: http://dx.doi.org/10.21037/jtd-2019-pitd-12).

\section{Methods}

\section{Objectives}

In this IRB approved retrospective single center clinical study to determine clinical utility of a follow-up cardiac nuclear stress test in patients following a coronary CT angiogram. We sought to determine clinical scenario where stress MPI is warranted and help find clinical conditions in which CCTA is sufficient to rule out coronary artery disease (CAD) as cause of patients' chest pain and nuclear stress MPI can be omitted.

\section{Patient population, data collection and clinical characteristics}

A total of 1,000 patients were evaluated in this study who presented with symptoms of ACS to the ED. Patients who had elevated troponin or abnormal ECG findings at initial presentation or prior to cardiac nuclear MPI were excluded from the study. All patients who underwent 64or 320-detector row ECG-gated CCTA as well as a followup nuclear MPI were included in the study. All patients included in the study underwent cardiac nuclear MPI within one month of the CCTA. Patients who had diagnostics studies limited by artifact [e.g., suboptimal intravenous (IV) contrast bolus in CCTA, motion artifact on CCTA or MPI, etc.] were excluded from the study. We identified 100 such patients and performed a chart review of the MPI results.

Patients' electronic medical record were reviewed between January 2005 and December 2013. All patients including in the study had chest pain as their chief complaint and all patients included in the study had no clinically relevant ECG findings, i.e., normal sinus rhythm without ST-segment or T-wave changes. Additionally, all patients had a normal point-of-care troponin test. Patient demographics including age, gender, and treatment history were reviewed.

Two experienced radiologists with reviewed the CCTA as well as the nuclear stress MPI. The readers were blinded to patients' clinical information. The radiologists interpreting the scans were aware of the objectives of the study.

\section{Statistical analysis}

Measures of central tendencies were performed. Paired $t$-test and Fischer exact test were performed to evaluate association between continuous and categorical variable. SPSS V.18 was utilized to perform statistical analysis.

\section{Results}

A total of 100 patients met the inclusion criteria, including normal ECG and cardiac troponin at initial presentation. 
Patient demographics include average age 64.3 [32-89] years, 59 male, 41 females. Ninety-five/100 patients had at least one vessel with $50-70 \%$ coronary artery diameter stenosis measured on CCTA. There were no focal perfusion abnormalities identified on cardiac nuclear MPI in patients with less than $70 \%$ stenosis diagnosed on CCTA (Figure 1). Five percent of patients were identified with coronary arterial narrowing greater than $70 \%$ on CCTA (Figure 2) and all 5 of these patients have evidence of abnormal cardiac nuclear stress test [e.g., stress-induced perfusion abnormalities (Figure 3), chest pain, abnormal ECG (Figure 2)]. Patients with positive nuclear stress test, CCTA detected obstructive stenosis $(>70 \%)$ in the left anterior descending artery $(4 / 5)$, left circumflex artery (3/5) and right coronary artery (2/5).

There was no statistically significant association between patients' age and positive stress MPI findings. Also, there was no statistically significant difference between patients' gender and positive stress MPI finding.

All of the patients with CCTA diagnosed coronary stenosis of less than $70 \%$, were discharged home following no significant perfusion deficits on MPI. There was no reported mortality on these patient 1-month after discharge from the hospital. Two of the five patients had greater than 90 percent luminal narrowing. These two patients underwent conventional coronary angiography, which confirmed high grade stenosis and were treated with angioplasty and stent placement. All five patients were followed at one month after discharge from hospital and there was no severe cardiac related adverse event reported within one-month after hospital discharge.

\section{Discussion}

CCTA is still not part of the standard of care (SOC) for diagnosis of CAD. However, American College of Cardiology Foundation (ACCF) and other subspecialty societies agree that CCTA can be used as a safe method to evaluate, diagnose and risk stratify patients with low or intermediate risk for $\mathrm{CAD}$, such as patients negative point of care ECG and troponin exams (10). There have been multiple clinical trials to evaluate the sensitivity and cost effectiveness of CCTA comparing to SOC for diagnosis of CAD. Hulten et al. in a meta-analysis study on four randomized clinical trials: 1,869 patients underwent CCTA diagnostic evaluation meanwhile 1,397 patients underwent SOC (5). Hulten et al. concluded: (I) CCTA is safe to evaluate low- to intermediate-risk patients presenting with chest pain; (II) the mortality rate, rate of MI, rate of return ED visits +/- recurrent hospitalization for cardiac etiologies is similar in the SOC and CCTA cohorts; (III) overall patient length of stay were reduced in patients triaged with CCTA; (IV) 3 out of 4 studies showed cost saving in CCTA group comparing to SOC group; (V) patients undergoing CCTA had a slight increased rate (2\%) of conventional coronary angiogram and revascularization compared with the SOC group. However, they were not able to determine whether this small difference was because of overuse of conventional angiogram and revascularization or underutilization in the SOC group (5).

A meta-analysis by Foy et al. in 2017 included 13 clinical trials: 10,315 patients underwent CCTA, meanwhile, 9,777 patients underwent cardiac nuclear stress MPI (9). These patients were clinically followed for approximately 18 months. There was no statistically significant difference between CCTA and cardiac nuclear MPI groups in the rate death or rate of cardiac-related hospitalization. However, it was noted that CCTA cohort had decreased incidence of MI. Interestingly, Foy et al. also demonstrated CCTA cohort was $2-3 \%$ more likely to undergo conventional coronary angiography and revascularization therapy. Additionally, the CCTA cohort was more likely to receive diagnosis of new $\mathrm{CAD}$ and to have initiated aspirin or statin therapy (9).

To evaluate downstream impact of CCTA-assisted diagnosis on conventional coronary angiography, Williams et al. conducted a prospective multicenter controlled trial. Four thousand one hundred and forty-six patients were randomized into two groups: SOC $v s$. SOC plus CCTA (11). Williams et al. demonstrated that in patient with CAD-related chest pain, CCTA led to "more appropriate" utilization of invasive angiography and preventative treatments to reduce risk of fatal and nonfatal ACS (11). Also, patients in the CCTA cohort who underwent conventional coronary angiography were more likely to have obstructive CAD than patients in the SOConly group (11).

These studies show that CCTA is more sensitive for CAD detection than cardiac nuclear MPI; it is not surprising that it causes greater downstream utilization of resources. A more sensitive diagnostic test is likely to identify a larger number of patients with disease leading to increase subsequent resource utilization in efforts to treat the underlying disease. Conversely, CCTA has a low false positive rate than cardiac nuclear MPI; which can potentially lead to decreased rate of cardiac catheterization in patients falsely diagnosed with CAD in the cardiac 




B

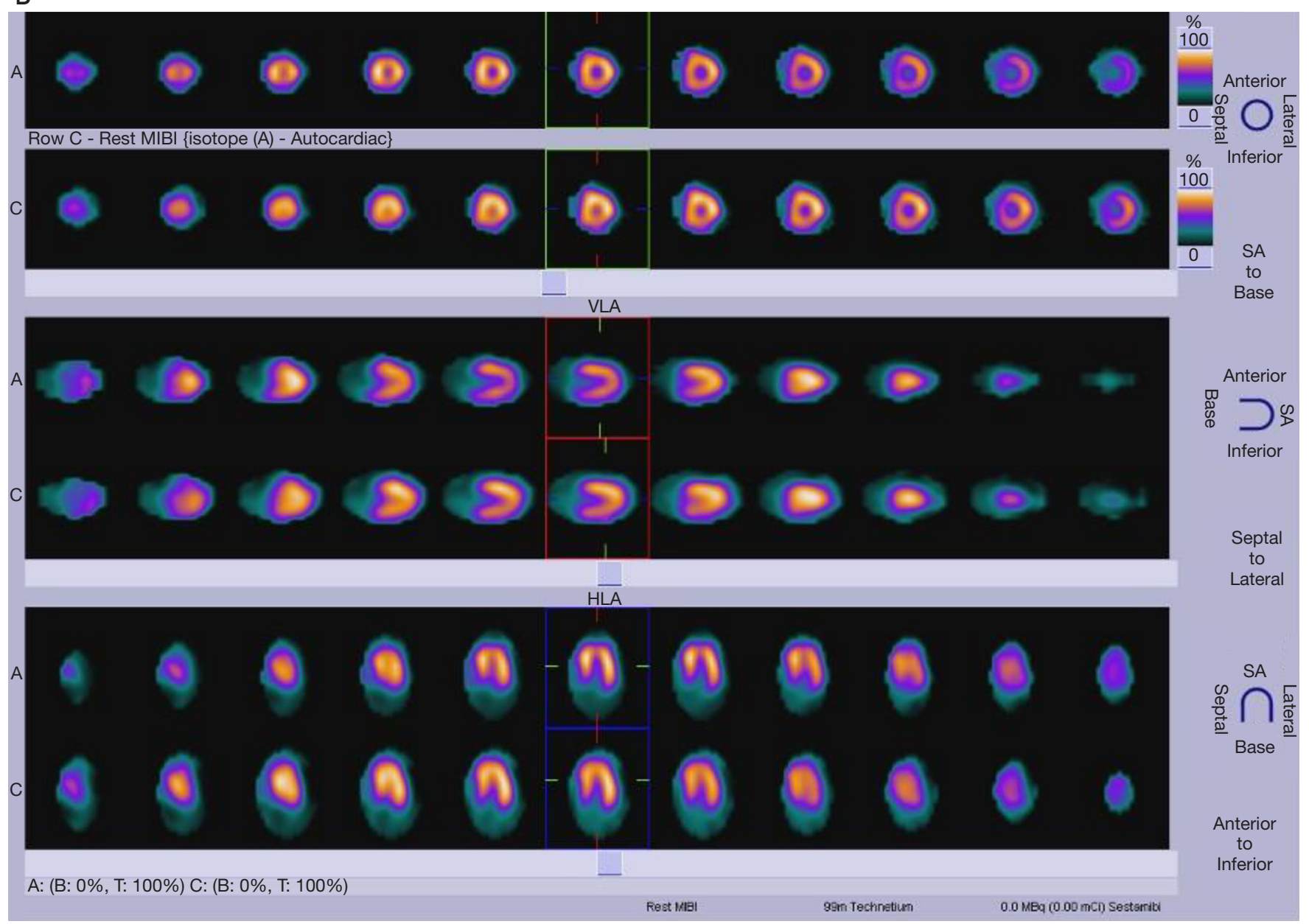




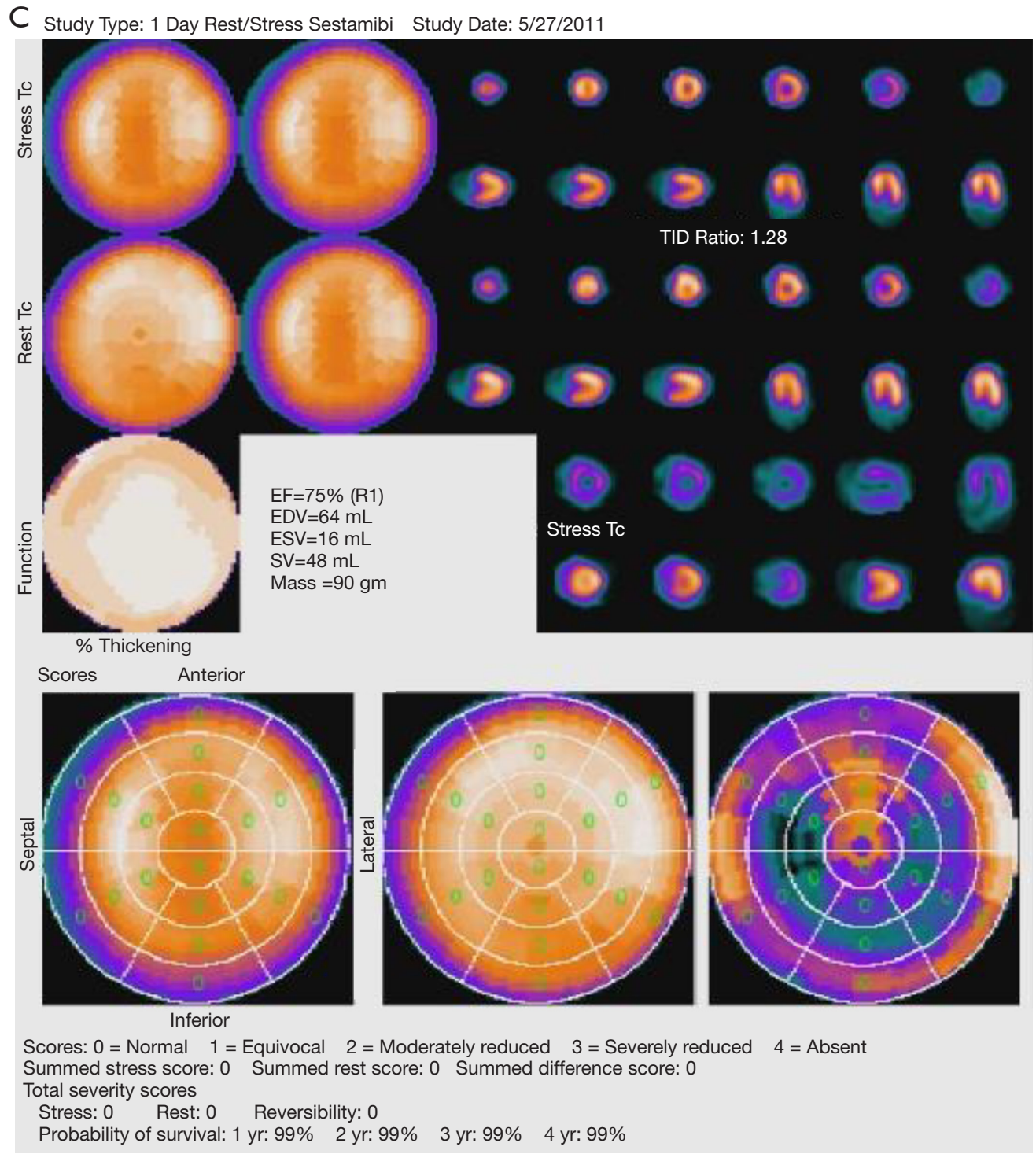

Figure 1 A 57-year-old male with chest pain underwent CCTA (A) which demonstrates calcified plaque in the LAD with 50\% stenosis. Agatston calcium score was 119 and MESA of 77 percentile. Nuclear stress MPI (B,C) are performed which demonstrates no significant perfusion abnormality. Patient was asymptomatic during rest and stress phase of the exam and no ECG changes were noted during rest or stress periods. SA, short-axis; MIBI, myocardial perfusion imaging test; VLA, vertical long axis; HLA, horizontal long axis; TID, transient ischemic dilation; EF, ejection fraction; EDV, end diastolic volume; ESV, end systolic volume; SV, stroke volume; MESA, multiethnic study of atherosclerosis; CCTA, coronary computed tomographic angiography; ECG, electrocardiogram; LAD, left anterior descending.

nuclear MPI (which is part of SOC) (12).

Our study showed that all patients who had severe coronary artery stenosis (more than $70 \%$ stenosis in one of the major coronary arteries) had a positive cardiac nuclear MPI requiring treatment. On the other hand, patients with mild to moderate coronary artery stenosis (less than $70 \%$ stenosis) did show normal stress test result. Our findings suggest that CCTA can not only reliably exclude severe CAD (as shown by numerous prior studies), but also that there is limited utility of performing a cardiac nuclear MPI in low-to-intermediate patients with CCTA showing mild to moderate stenosis ( $<70 \%$ vessel diameter). Our study also further supports the fact that CCTA can not only help rule out ACS but also help shorten duration of patient 

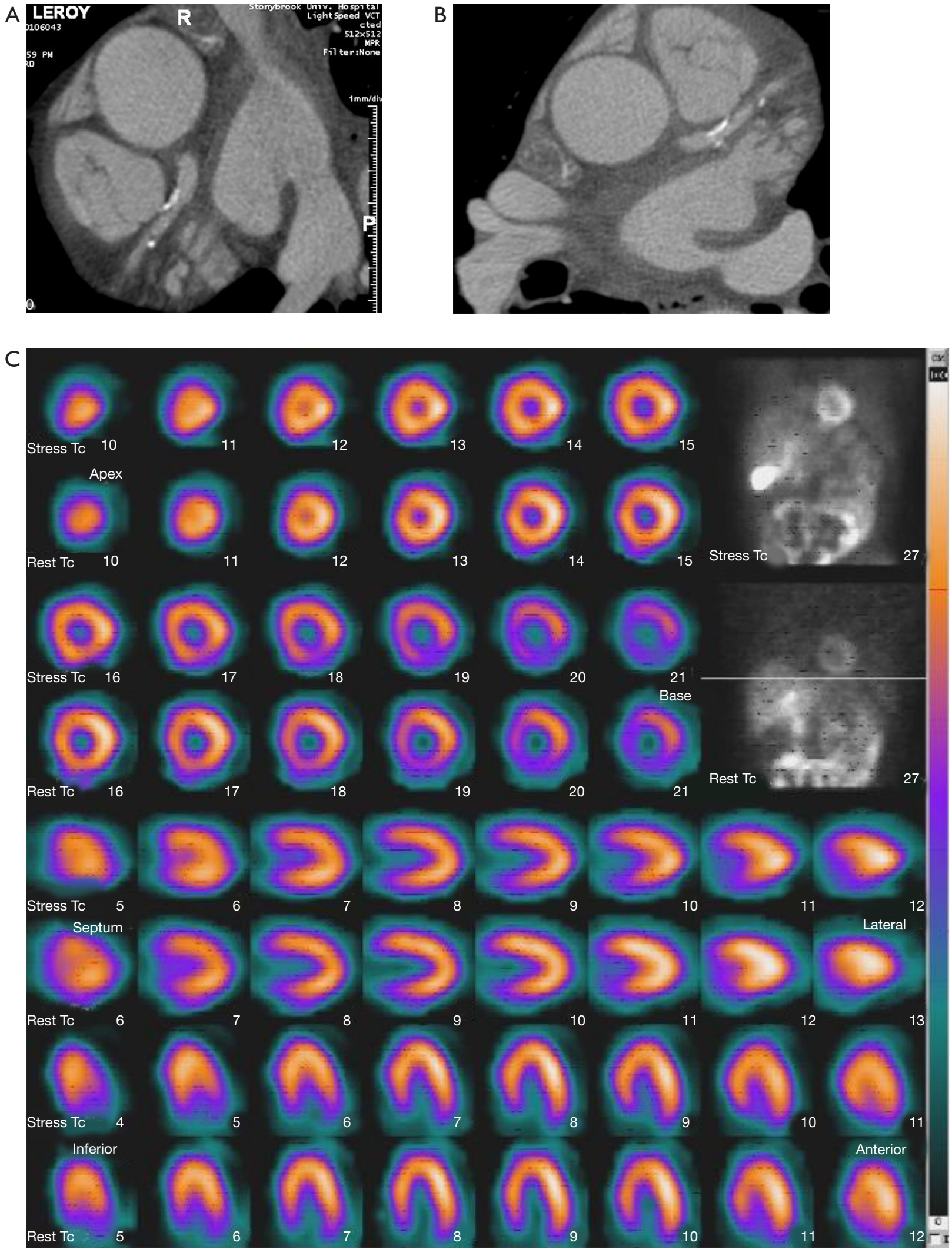


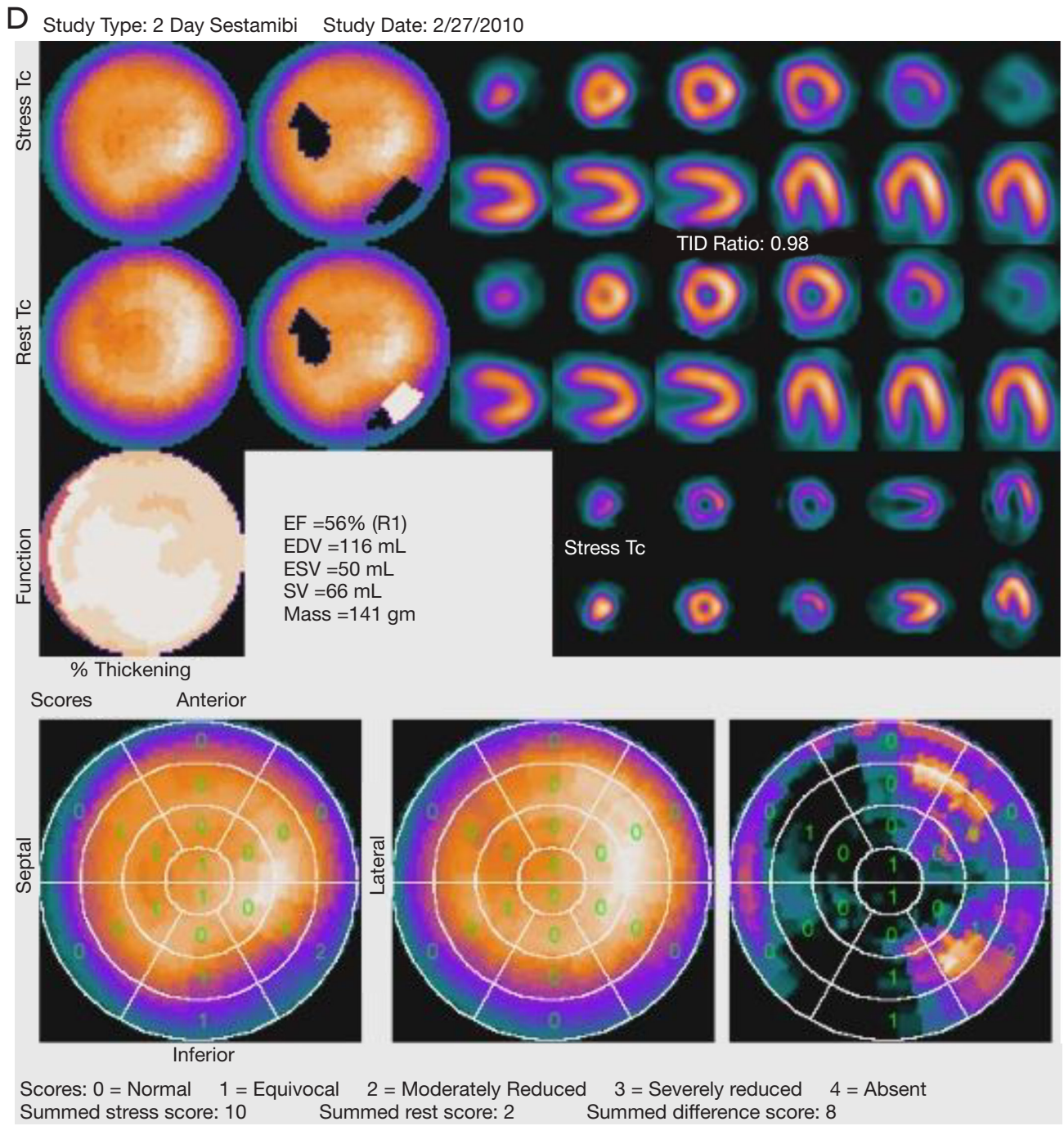

Figure 2 A 61-year-old male with history of hypertension presented to the emergency department with acute chest pain. The CCTA $(\mathrm{A}, \mathrm{B})$ demonstrates mixed plaque with approximately $75 \%$ stenosis of the proximal LAD. The Agatston calcium score was 518; MESA $70^{\text {th }}$ percentile. (C,D) Nuclear MPI performed to evaluate for infarction and/or ischemia. Although the images at rest (C) show there is no significant perfusion defect to suggest ischemia or infarction, stress images (D) show stress-induced ST depression on ECG in leads V4-V6 in the expected distribution of the LAD. LAD, left anterior descending; ECG, electrocardiogram; CCTA, coronary computed tomographic angiography; MPI, myocardial perfusion imaging; MESA, multiethnic study of atherosclerosis; EF, ejection fraction; EDV, end diastolic volume; ESV, end systolic volume; SV, stroke volume.

visit in the ED (5-11). The high prevalence of helical CT scanners and institution of CCTA for work-up of chest pain can potentially identify high risk patients in the ED in a short period of time. Patients can be divided into two groups: group of patients with severe coronary artery stenosis who should stay at the hospital and undergo cardiac catheterization and second group with mild to moderate coronary artery stenosis who can be discharged from ED for outpatient follow-up without further testing (e.g., cardiac nuclear MPI). This approach will dramatically decrease the length of stay in patient with low to intermediate risk for ACS.

Although our study showed promising data regarding the use of CCTA, there are some notable limitations of our study. These include a retrospective study design leading to inherent bias in sample selection. Although patients' outcome is evaluated for up to 30 days after 

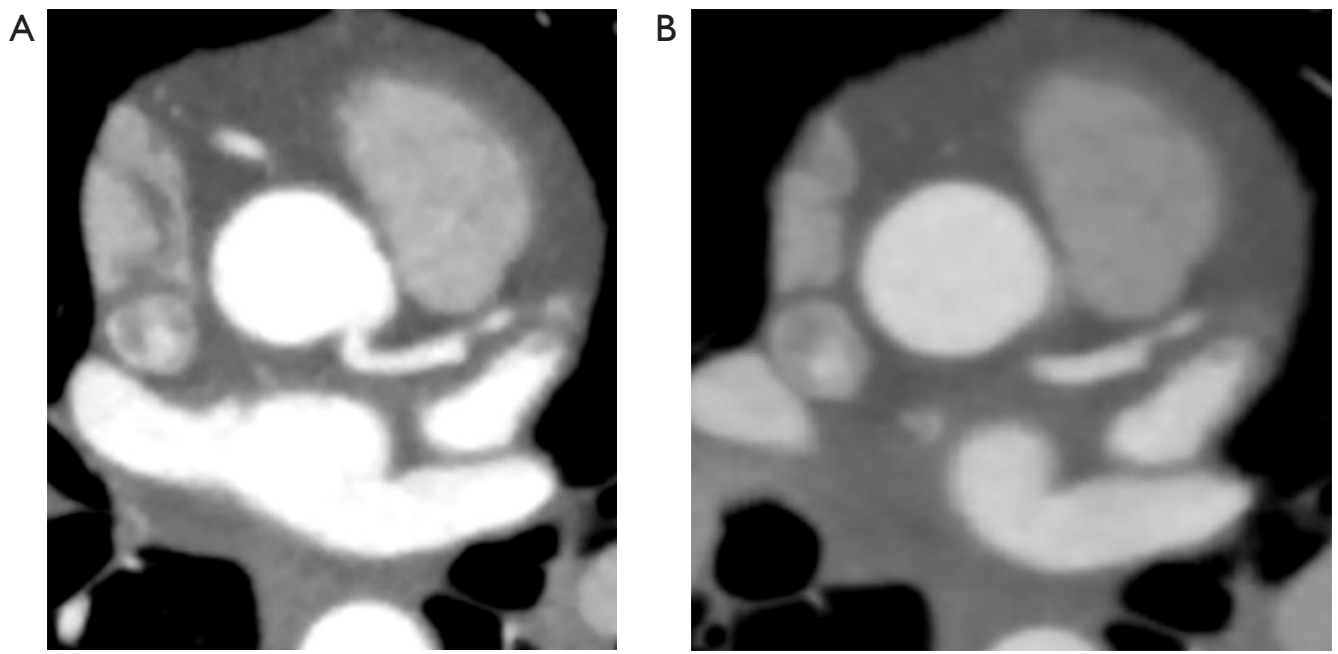

C
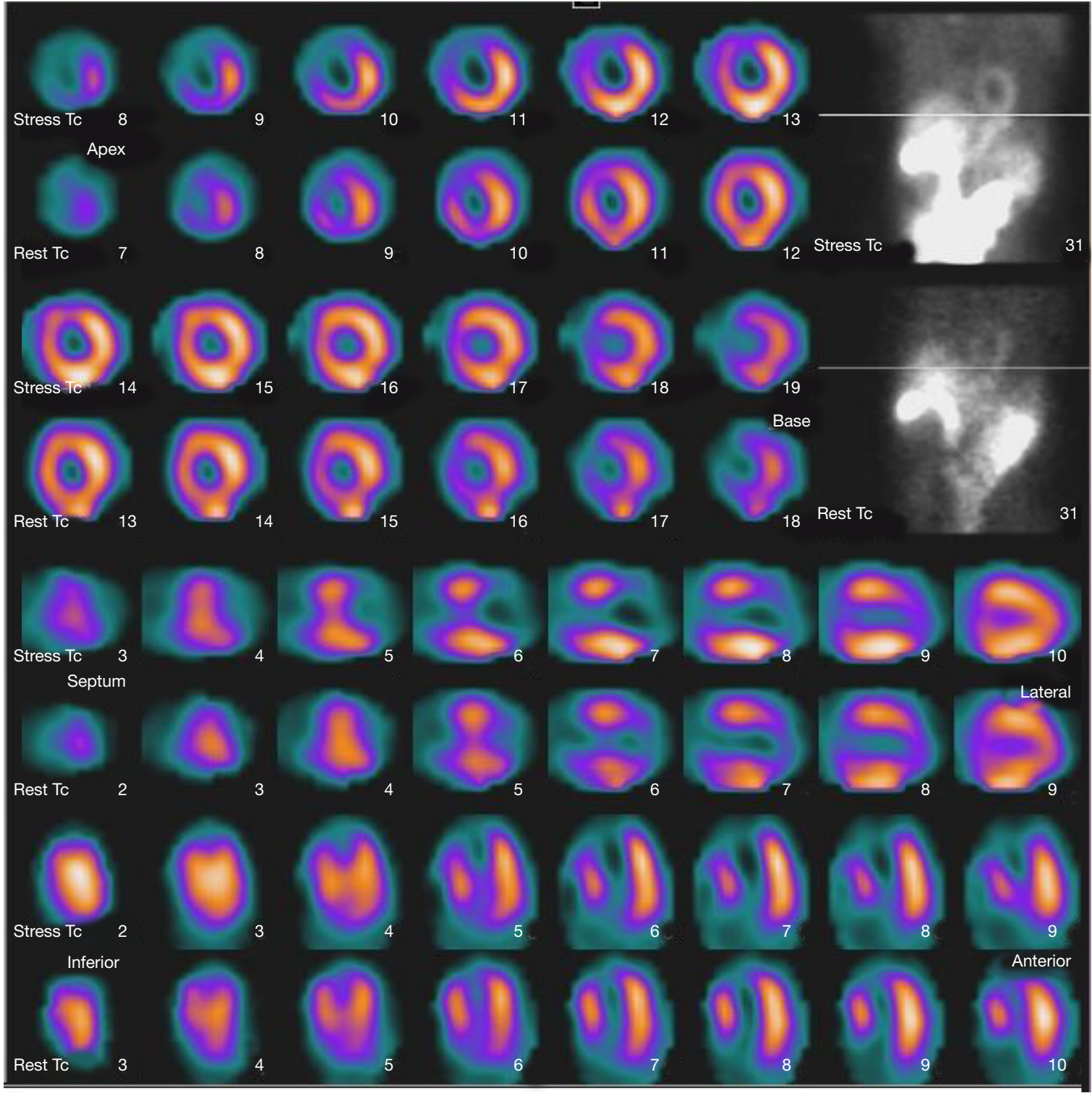


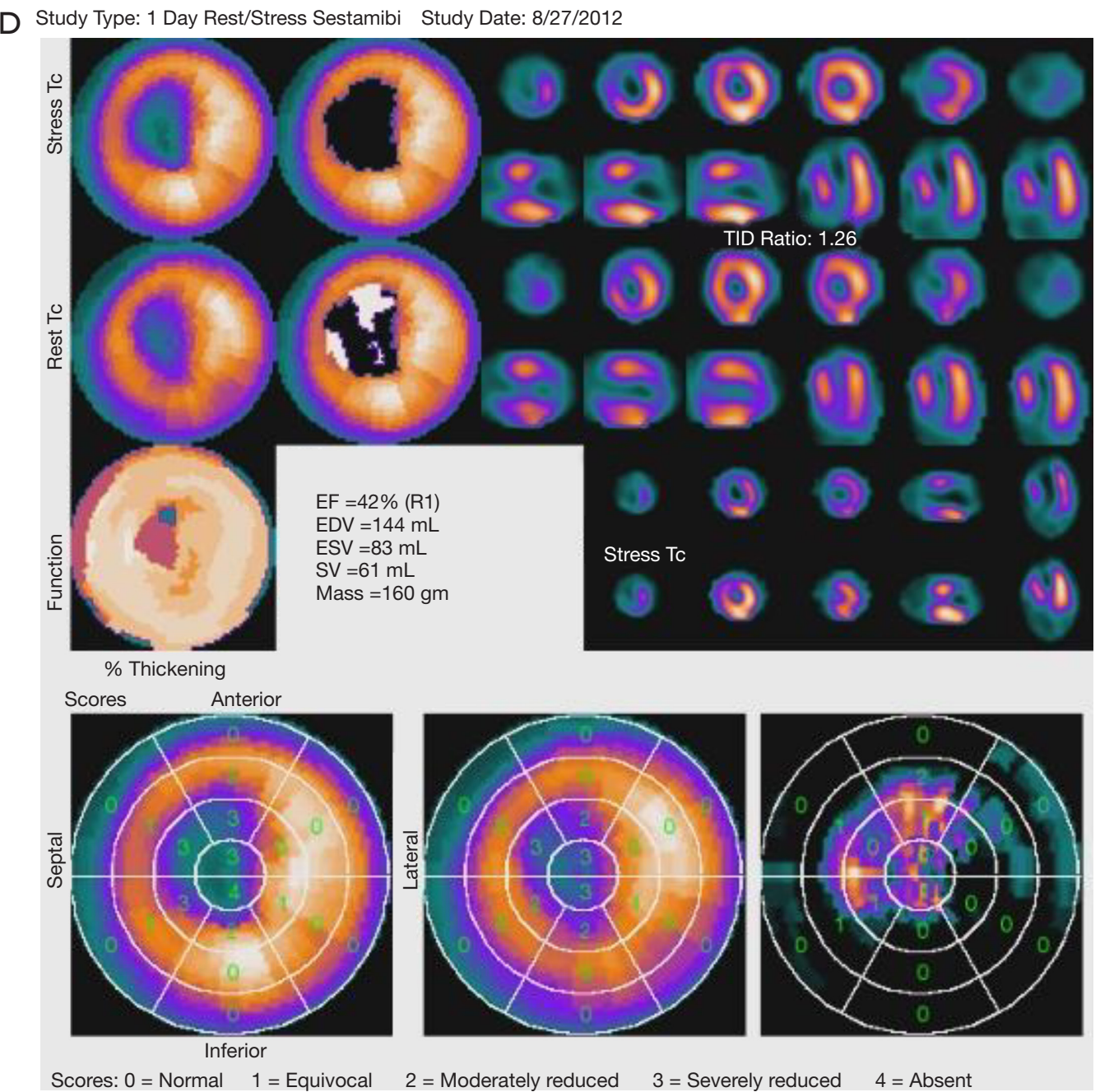

Figure 3 A 55 -year-old female presented to the ED with left arm pain. The CCTA (A,B) performed demonstrates mixed predominantly soft plaque in the prox-mid LAD with $>90 \%$ stenosis. The Agatston calcium score was 8 with MESA of 86 percentile. Cardiac nuclear MPI (C,D) revealed severe mainly fixed perfusion abnormality with mild to moderate reversibility in the anterior, anteroseptal and apical segments. Also, the LVEF is reduced (42\%). EF, ejection fraction; EDV, end diastolic volume; ESV, end systolic volume; SV, stroke volume; ED, emergency department; CCTA, coronary computed tomographic angiography; LAD, left anterior descending; MESA, multiethnic study of atherosclerosis; MPI, myocardial perfusion imaging; LVEF, left ventricular ejection fraction.

hospital discharged, detailed clinical assessment of patient post-hospital discharge is scarce in patients' electronic medical records. Additionally, small sample size weakens the statistical inferences drawn from this study. As such, prospective larger studies are required to validate our study findings. Future studies should evaluate whether downstream over utilization of conventional coronary angiography in CCTA is associated with the better clinical outcome and overall reduction of cost of care in the long term in this patient population.

\section{Conclusions}

In low-to-intermediate risk patients with chest pain and evidence of non-critical coronary artery stenosis (less than $70 \%$ diameter) diagnosed on CCTA, a follow-up cardiac nuclear perfusion imaging is of limited value. CCTA is a sensitive method with high negative predictive value that can be used in the ED as an accurate tool to identify patients with coronary artery stenosis amongst low to intermediate ACS risk patients. 


\section{Acknowledgments}

We would like to thank Javod Savoj, internal medicine resident at Riverside Community Hospital for his assistance with review of literature.

Funding: This work was supported by the National Cancer Institute of the National Institutes of Health under grant: 5K12CA001727.

\section{Footnote}

Provenance and Peer Review: This article was commissioned by the Guest Editor (Ammar Chaudhry) for the series "Role of Precision Imaging in Thoracic Disease" published in fournal of Thoracic Disease. The article was sent for external peer review organized by the Guest Editor and the editorial office.

Reporting Checklist: The authors have completed the STROBE guideline checklist. Available at http://dx.doi. org/10.21037/jtd-2019-pitd-12

Conflicts of Interest: All authors have completed the ICMJE uniform disclosure form (available at: http://dx.doi. org/10.21037/jtd-2019-pitd-12). The series "Role of Precision Imaging in Thoracic Disease" was commissioned by the editorial office without any funding or sponsorship. AC served as an unpaid Guest Editor of the series. The other authors have no conflicts of interest to declare.

Ethical Statement: The authors are accountable for all aspects of the work in ensuring that questions related to the accuracy or integrity of any part of the work are appropriately investigated and resolved. The study was conducted in accordance with the Declaration of Helsinki (as revised in 2013). The study was approved by the institutional review board (No. SB561506-01) and written informed consent was obtained from all patients.

Open Access Statement: This is an Open Access article distributed in accordance with the Creative Commons Attribution-NonCommercial-NoDerivs 4.0 International License (CC BY-NC-ND 4.0), which permits the noncommercial replication and distribution of the article with the strict proviso that no changes or edits are made and the original work is properly cited (including links to both the formal publication through the relevant DOI and the license). See: https://creativecommons.org/licenses/by-nc-nd/4.0/.

\section{References}

1. Hing E, Hall MJ, Xu J. National Hospital Ambulatory Medical Care Survey: 2006 outpatient department summary. Natl Health Stat Report 2008;(4):1-31.

2. Lee TH, Rouan GW, Weisberg MC, et al. Clinical characteristics and natural history of patients with acute myocardial infarction sent home from the emergency room. Am J Cardiol 1987;60:219-24.

3. Pope JH, Aufderheide TP, Ruthazer R, et al. Missed diagnoses of acute cardiac ischemia in the emergency department. N Engl J Med 2000;342:1163-70.

4. Klocke FJ, Baird MG, Lorell BH, et al. ACC/AHA/ASNC guidelines for the clinical use of cardiac radionuclide imaging--executive summary: a report of the American College of Cardiology/American Heart Association Task Force on Practice Guidelines (ACC/AHA/ASNC Committee to Revise the 1995 Guidelines for the Clinical Use of Cardiac Radionuclide Imaging). J Am Coll Cardiol 2003;42:1318-33.

5. Hulten E, Pickett C, Bittencourt MS, et al. Outcomes after coronary computed tomography angiography in the emergency department: a systematic review and metaanalysis of randomized, controlled trials. J Am Coll Cardiol 2013;61:880-92.

6. Iskandrian AE, Bateman TM, Belardinelli L, et al. Adenosine versus regadenoson comparative evaluation in myocardial perfusion imaging: results of the ADVANCE phase 3 multicenter international trial. J Nucl Cardiol 2007; 14:645-58.

7. Hamilton-Craig C, Fifoot A, Hansen M, et al. Diagnostic performance and cost of $\mathrm{CT}$ angiography versus stress ECG--a randomized prospective study of suspected acute coronary syndrome chest pain in the emergency department (CT-COMPARE). Int J Cardiol 2014;177:867-73.

8. Goldstein JA, Chinnaiyan KM, Abidov A, et al. The CTSTAT (Coronary Computed Tomographic Angiography for Systematic Triage of Acute Chest Pain Patients to Treatment) trial. J Am Coll Cardiol 2011;58:1414-22.

9. Foy AJ, Dhruva SS, Peterson B, et al. Coronary Computed Tomography Angiography vs Functional Stress Testing for Patients With Suspected Coronary Artery Disease: A Systematic Review and Meta-analysis. JAMA Intern Med 2017;177:1623-31.

10. Taylor AJ, Cerqueira M, Hodgson JM, et al. ACCF/ SCCT/ACR/AHA/ASE/ASNC/NASCI/SCAI/SCMR 
2010 Appropriate Use Criteria for Cardiac Computed Tomography. A Report of the American College of Cardiology Foundation Appropriate Use Criteria Task Force, the Society of Cardiovascular Computed Tomography, the American College of Radiology, the American Heart Association, the American Society of Echocardiography, the American Society of Nuclear Cardiology, the North American Society for Cardiovascular Imaging, the Society for Cardiovascular Angiography and Interventions, and the Society for

Cite this article as: Gul M, Sheikh M, Chaudhry A, Gerges L, Al Halabi H, Feldman E, Chaudhry A. Role of cardiac nuclear stress perfusion exam after computed tomographic coronary angiogram for evaluation of obstructive coronary artery disease in patients with chest pain. J Thorac Dis 2020;12(9):5067-5077. doi: 10.21037/jtd-2019-pitd-12
Cardiovascular Magnetic Resonance. J Cardiovasc Comput Tomogr 2010;4:407.e1-33.

11. Williams MC, Hunter A, Shah ASV, et al. Use of Coronary Computed Tomographic Angiography to Guide Management of Patients With Coronary Disease. J Am Coll Cardiol 2016;67:1759-68.

12. Rahsepar AA, Arbab-Zadeh A. Cardiac CT vs. Stress Testing in Patients with Suspected Coronary Artery Disease: Review and Expert Recommendations. Curr Cardiovasc Imaging Rep 2015;8:29. 